\title{
Hydrocephalus after arteriovenous malformation rupture
}

\author{
Bradley A. Gross, M.D., Pui Man Rosalind Lai, B.A., and Rose Du, M.D., Ph.D. \\ Department of Neurological Surgery, Brigham and Women's Hospital and Harvard Medical School, Boston, \\ Massachusetts
}

\begin{abstract}
Object. The rates and risk factors for external ventricular drain (EVD) placement and long-term shunt dependence in patients with ruptured arteriovenous malformations (AVMs) have not been systematically studied. In this study the authors evaluated the rates of EVD placement and shunt dependence, and risk factors for them, in a cohort of patients with ruptured AVMs.

Methods. The records of 87 consecutive patients with ruptured AVMs were reviewed for patient demographics, hemorrhage pattern, AVM angioarchitectural features, and surgical treatment. Univariate and multivariate logistic regression analyses were performed to evaluate risk factors for EVD placement, permanent shunt dependence, and long-term outcome (as measured by the modified Rankin Scale).

Results. Thirty-eight patients (44\%) required EVD placement, and $16(18 \%)$ required a permanent shunt. Statistically significant risk factors for EVD placement in the univariate analysis included initial Glasgow Coma Scale (GCS) score $(\mathrm{p}=0.002)$, the presence of intraventricular hemorrhage (IVH; $\mathrm{p}<0.001)$, AVM-associated aneurysms $(p=0.002)$, and early surgery $(p=0.01)$. Multivariate analysis revealed only AVM-associated aneurysms as statistically significant $(p=0.006)$. Risk factors for shunt placement included initial GCS score $(p=0.003)$, IVH $(p=0.01)$, deep supratentorial location $(\mathrm{p}=0.034)$, and associated aneurysms $(\mathrm{p}=0.03)$. Multivariate analysis revealed initial GCS score as a statistically significant risk factor $(\mathrm{p}=0.041)$ as well as a strong trend for associated aneurysms $(\mathrm{p}=$ 0.06). Patient age, sex, associated subarachnoid hemorrhage, AVM grade, AVM size, and deep venous drainage were not associated with EVD placement or long-term shunt dependence.

Conclusions. Hydrocephalus from AVM rupture was associated with initial GCS score, IVH, and AVM-associated aneurysms. Arteriovenous malformations with associated aneurysms thus not only have a greater risk of hemorrhage but also a greater risk of hemorrhage-associated morbidity as a result of hydrocephalus. (http://thejns.org/doi/abs/10.3171/2013.2.FOCUS12368)
\end{abstract}

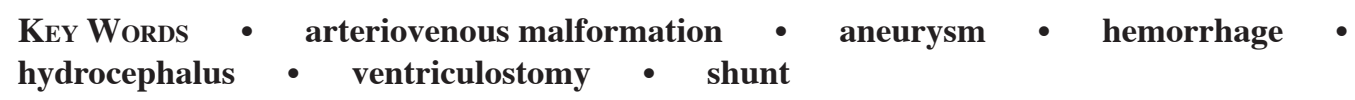

A PPROXIMATELY $50 \%$ of patients with AVMs will present with hemorrhage, ${ }^{12}$ resulting in morbidity in $30 \%-50 \%$ of cases and death in $10 \%$ of cases. ${ }^{1,3,7,8}$ Although hemorrhage is the most common presentation modality among patients with AVMs, $7,8,12$ hydrocephalus after AVM rupture, evaluated in the form of EVD placement and long-term shunt dependence, has not been well studied. In this study we evaluated the rates of EVD placement and shunt dependence, and risk factors for them, in a cohort of patients with ruptured AVMs.

\section{Methods}

With approval from the institutional review board of Brigham and Women's Hospital, the records of a consec-

\footnotetext{
Abbreviations used in this paper: AVM = arteriovenous malformation; EVD = external ventricular drain; GCS = Glasgow Coma Scale; IVH = intraventricular hemorrhage; $\mathrm{mRS}=$ modified Rankin Scale; $\mathrm{SAH}=$ subarachnoid hemorrhage.
}

utive series of patients with AVMs who were evaluated between January 2005 and November 2012 at our institution were reviewed. Patients with dural arteriovenous shunts, spinal arteriovenous shunts, and cavernous malformations were excluded, along with those with hemorrhagic AVMs presenting with lethal, massive hemorrhage (GCS score of 3 and bilateral fixed and dilated pupils with no intervention performed).

\section{Data Extraction}

From a detailed chart review, the following information was extracted: patient age, sex, initial GCS score, hemorrhage pattern (intraparenchymal, subarachnoid, and/ or intraventricular), AVM Spetzler-Martin grade (size, location, drainage),$^{11}$ associated aneurysms, and whether the patient underwent early surgery to treat the AVM and/or evacuate the hemorrhage (within 2 weeks). Trace SAH in a single convexity sulcus was not included as a formal subarachnoid bleed for our analysis.

Our criteria for EVD placement mirror those for pa- 
tients with aneurysmal SAH-nonpharmacological drowsiness and/or obtundation as a manifestation of hydrocephalus. The presence of headache or intraventricular blood were not, in themselves, indications for CSF diversion. We noted the frequency of EVD placement and resultant complications (infection, hemorrhage), permanent shunt dependence, and long-term outcome (measured as the patient's mRS score at follow-up).

\section{Statistical Analysis}

The statistical program Stata version 12.0 was used to study potential risk factors for EVD placement and long-term shunt dependence. In addition, the impact of these factors on long-term follow-up $\mathrm{mRS}$ score was also evaluated. Univariate linear regressions were performed for the following factors: patient age, sex, initial GCS score, the presence of SAH, IVH, AVM grade, nidus size, deep venous drainage, AVM location (superficial vs deep supratentorial vs infratentorial), associated aneurysms, and early surgery (within 2 weeks). Subsequent multivariate linear regressions were performed, incorporating any factors nearing significance $(\mathrm{p}<0.2)$, using patient age, sex, and initial GCS score empirically as covariates.

\section{Results}

\section{Patient Characteristics}

Of 142 patients with AVMs, 87 (61\%) presented with hemorrhage. None of the 55 patients with unruptured AVMs required CSF diversion due to obstructive hydrocephalus or other pathophysiology associated with their vascular malformation. The remaining cohort of 87 patients with ruptured AVMs had a mean age of $46 \pm 17.4$ years. The male to female ratio was 1.2:1. Of 87 hemorrhages, 73 (84\%) had an intraparenchymal component, $48(55 \%)$ an intraventricular component, and $13(15 \%)$ a subarachnoid component. Fifty-nine AVMs (68\%) were supratentorial and superficial, 12 (14\%) were supratentorial and deep, and $16(18 \%)$ were infratentorial. The mean nidus size was $2.4 \pm 1.5 \mathrm{~cm}$. Thirty-three AVMs $(38 \%)$ had associated deep venous drainage, and 23 (26\%) had associated feeding artery or angiographically visualized intranidal aneurysms. The mean Spetzler-Martin grade was $2.3 \pm 1.0$. Fifty-eight patients $(67 \%)$ underwent early surgical treatment of their AVM along with evacuation of their intraparenchymal hematoma, if present.

Thirty-eight patients (44\%) required placement of an EVD, and 16 required permanent CSF diversion (18\% of entire cohort, $42 \%$ of those undergoing initial ventriculostomy). Of 38 cases with ventriculostomy placement, $4(11 \%)$ resulted in asymptomatic intraparenchymal hematomas, and $3(8 \%)$ became infected. No symptomatic hemorrhages occurred, and no infectious or hemorrhagic complications occurred after the 16 permanent shunt procedures.

\section{Univariate and Multivariate Analysis}

Univariate analysis of risk factors for EVD placement revealed GCS score (OR 0.86, 95\% CI 0.78-0.95, $\mathrm{p}=0.002)$, IVH (OR 6.46, 95\% CI 2.44-17.1, p < 0.001), associated aneurysms (OR 4.86, 95\% CI 1.74-13.53, p = 0.002 ), and early surgery (OR 2.22, 95\% CI 0.87-5.69, $\mathrm{p}=0.01$ ) to be statistically significant (Table 1). Patient age, sex, associated SAH, AVM grade, AVM size, deep venous drainage, and AVM location were not significant. Multivariate analysis revealed only associated aneurysms to be a statistically significant risk factor for EVD placement (OR 20.96, 95\% CI 2.41-181.9, p = 0.006).

Statistically significant risk factors for shunt placement included initial GCS score (OR 0.84, 95\% CI 0.75$0.94, p=0.003)$, IVH (OR 7.62, 95\% CI 1.61-36, $p=0.01)$, deep supratentorial location (OR 4.16, 95\% CI 1.12-15.46, $\mathrm{p}=0.034$ ), and associated aneurysms (OR 3.44, 95\% CI $1.11-10.6, p=0.03$; Table 1) Patients with ruptured, supratentorial, superficial AVMs had a lower risk of shunt dependence (OR 0.28, 95\% CI 0.093-0.87, p = 0.028). Patient age, sex, associated SAH, AVM grade, AVM size, deep venous drainage, and early surgery were not associated with long-term shunt dependence. Multivariate analysis revealed initial GCS score to be a statistically significant risk factor (OR 0.76, 95\% CI 0.59-0.99, p = 0.041 ) as well as a strong trend for associated aneurysms (OR 6.31, 95\% CI 0.93-43.1, p = 0.06).

\section{Impact on $m R S$ Score}

A univariate and multivariate analysis was performed to evaluate the potential impact of each risk factor on follow-up mRS score (Table 2). Shunt placement was also included as a means to evaluate its relationship to long-term outcome. Statistically significant risk factors from univariate analysis were increasing age $(\mathrm{p}<0.001)$, lower initial GCS score $(\mathrm{p}<0.001)$, and the placement of a shunt $(\mathrm{p}=0.022)$. Multivariate analysis continued to demonstrate older age as a significant risk factor $(\mathrm{p}=$ $0.02)$, and a trend for the placement of a shunt $(\mathrm{p}=0.26)$.

\section{Discussion}

Cerebral AVMs are a considerable source of neurological morbidity as a result of hemorrhages, seizures, and/or steal phenomena., ${ }^{1,6,12}$ In contrast to aneurysm rupture in which morbidity and mortality are largely attributable to hydrocephalus and infrequently from local mass effect on eloquent parenchyma from intraparenchymal hemorrhage, both mechanisms are at play with AVM rupture. Prospective studies evaluating AVM rupture generally cite annual rates from $2.4 \%$ to $4.6 \%$, with prior hemorrhage consistently serving as a significant risk factor for hemorrhage along with deep location, deep venous drainage, and associated aneurysms. ${ }^{4,6,12}$ A systematic approach to evaluating morbidity from AVM hemorrhage, requiring a stratification of morbidity resulting from hydrocephalus and morbidity resulting from local mass effect of hemorrhage on eloquent parenchyma, has not been conducted.

This analysis quantifies the frequency of hydrocephalus after AVM rupture and risk factors for its occurrence. Overall, $44 \%$ of patients required the placement of an EVD and 18\% required permanent ventriculoperitoneal shunt placement. Intuitively, initial GCS score and the presence of IVH were significant risk factors for EVD 
Arteriovenous malformations and hydrocephalus

TABLE 1: Univariate analysis of risk factors for EVD placement and shunt placement*

\begin{tabular}{lcccc}
\hline \multicolumn{1}{c}{ Risk Factor } & EVD Placement OR $(95 \% \mathrm{Cl})$ & $\mathrm{p} \mathrm{Value}$ & Shunt Placement OR $(95 \% \mathrm{Cl})$ & $\mathrm{p} \mathrm{Value}$ \\
\hline age & $1.01(0.98-1.03)$ & 0.45 & $1.02(0.98-1.05)$ & 0.34 \\
female sex & $1.75(0.74-4.14)$ & 0.20 & $2.41(0.79-7.38)$ & 0.12 \\
initial GCS score & $0.86(0.78-0.95)$ & 0.002 & $0.84(0.75-0.94)$ & 0.003 \\
SAH & $0.78(0.23-2.60)$ & 0.68 & $1.41(0.35-5.84)$ & 0.64 \\
IVH & $6.46(2.44-17.1)$ & $<0.001$ & $7.62(1.61-36.0)$ & 0.01 \\
Spetzler-Martin grade & $1.11(0.73-1.68)$ & 0.62 & $1.21(0.72-2.05)$ & 0.47 \\
nidus size & $1.17(0.89-1.53)$ & 0.27 & $0.96(0.67-1.36)$ & 0.80 \\
deep venous drainage & $1.12(0.47-2.69)$ & 0.79 & $0.98(0.32-3.0)$ & 0.97 \\
superficial supratentorial & $0.55(0.22-1.37)$ & 0.20 & $0.28(0.093-0.87)$ & $\mathbf{0 . 0 3}$ \\
deep supratentorial & $1.34(0.40-4.55)$ & 0.64 & $4.16(1.12-15.46)$ & $\mathbf{0 . 0 3}$ \\
infratentorial & $1.86(0.62-5.57)$ & 0.27 & $1.64(0.45-5.96)$ & 0.45 \\
associated aneurysms & $4.86(1.74-13.5)$ & 0.002 & $3.44(1.11-10.6)$ & 0.03 \\
early surgery & $2.22(0.87-5.69)$ & 0.01 & $1.63(0.48-5.59)$ & 0.44 \\
\hline
\end{tabular}

* Statistically significant values $(p<0.05)$ are shown in boldface.

placement. Importantly, the presence of associated aneurysms was also a significant risk factor on both univariate and subsequent multivariate analysis. Initial GCS score and IVH likely no longer met statistical significance in the repeated multivariate analysis due to their high association with AVM-associated aneurysms. Sixteen (70\%) of 23 patients with associated aneurysms had IVH as compared with $32(50 \%)$ of 64 patients without aneurysms (p $=0.05$ ), and the mean initial GCS score for patients with associated aneurysms was 10.0 as compared with 11.4 for those without $(\mathrm{p}=0.22)$.

Initial GCS score, IVH, and associated aneurysms were also significant factors influencing shunt dependence as they were for EVD placement, with the addition of supratentorial deep AVMs. On multivariate analysis,

TABLE 2: Univariate analysis of factors potentially impacting long-term $\mathrm{mRS}$ score*

\begin{tabular}{lcc}
\hline \multicolumn{1}{c}{ Risk Factor } & Impact on mRS $\dagger$ & $p$ Value \\
\hline age & +0.042 & $<0.001$ \\
female sex & +0.52 & 0.19 \\
initial GCS score & -0.147 & $<0.001$ \\
SAH & +0.41 & 0.49 \\
IVH & +0.60 & 0.13 \\
Spetzler-Martin grade & +0.26 & 0.20 \\
nidus size & +0.25 & 0.054 \\
deep venous drainage & -0.29 & 0.48 \\
superficial supratentorial & -0.23 & 0.59 \\
deep supratentorial & -0.63 & 0.26 \\
infratentorial & +0.95 & 0.077 \\
associated aneurysms & +0.79 & 0.064 \\
early surgery & -0.14 & 0.75 \\
permanent shunt & +1.1 & 0.022 \\
\hline
\end{tabular}

* A higher mRS score represents a worse outcome.

$\dagger$ Regression coefficients. however, only initial GCS score continued to be a significant risk factor, with a strong trend for AVMs with associated aneurysms $(p=0.06)$. Other AVM morphological factors (size, location, and drainage) were not associated with the risk of hydrocephalus.

The rate of long-term shunt dependence from AVM rupture in this study (18\%) is comparable to the rates found in several studies evaluating hydrocephalus after aneurysm rupture. ${ }^{5,9}$ These studies primarily focused on a comparison of the risk of shunt dependence after coiling as compared with clipping of ruptured aneurysms and demonstrated no significant difference. ${ }^{5,9}$ In a somewhat analogous fashion, in this study early surgery was not associated with a lower risk of shunt dependence. The interesting finding in our study that associated aneurysms increase this risk has important therapeutic implications because it suggests that rupture from these AVMs may be more morbid. This data adds to the current literature that already reinforces the fact that the risk of hemorrhage is higher for these lesions. ${ }^{2,4,10}$ Taken together, these findings encourage the timely treatment of these AVMs to not only combat the greater risk of hemorrhage from these lesions, but also potentially greater associated morbidity from hemorrhage. Importantly, our final analysis reinforced the fact that hydrocephalus and shunt placement after AVM rupture has significant clinical implications, as shunt dependence was associated with worse longterm outcome as measured by mRS score.

\section{Conclusions}

The overall rate of early EVD placement was $44 \%$ and the rate of shunt dependence was 18\% after AVM rupture. Significant risk factors for both were initial GCS score, IVH, and associated aneurysms. Arteriovenous malformations with associated aneurysms thus not only have a greater risk of rupture but also a greater potential risk of permanent morbidity from rupture as a result of hydrocephalus, encouraging their timely treatment. 


\section{Disclosure}

The authors report no conflict of interest concerning the materials or methods used in this study or the findings specified in this paper.

Author contributions to the study and manuscript preparation include the following. Conception and design: Du, Gross. Acquisition of data: Gross, Lai. Analysis and interpretation of data: all authors. Drafting the article: Gross. Critically revising the article: all authors. Reviewed submitted version of manuscript: all authors. Approved the final version of the manuscript on behalf of all authors: Du. Statistical analysis: Lai. Administrative/technical/material support: Du. Study supervision: Du.

\section{References}

1. Brown RD Jr, Wiebers DO, Forbes G, O'Fallon WM, Piepgras DG, Marsh WR, et al: The natural history of unruptured intracranial arteriovenous malformations. J Neurosurg 68:352357, 1988

2. Brown RD Jr, Wiebers DO, Forbes GS: Unruptured intracranial aneurysms and arteriovenous malformations: frequency of intracranial hemorrhage and relationship of lesions. J Neurosurg 73:859-863, 1990

3. Crawford PM, West CR, Chadwick DW, Shaw MD: Arteriovenous malformations of the brain: natural history in unoperated patients. J Neurol Neurosurg Psychiatry 49:1-10, 1986

4. da Costa L, Wallace MC, Ter Brugge KG, O'Kelly C, Willinsky RA, Tymianski M: The natural history and predictive features of hemorrhage from brain arteriovenous malformations. Stroke 40:100-105, 2009

5. Dehdashti AR, Rilliet B, Rufenacht DA, de Tribolet N: Shuntdependent hydrocephalus after rupture of intracranial aneurysms: a prospective study of the influence of treatment modality. J Neurosurg 101:402-407, 2004
6. Hernesniemi JA, Dashti R, Juvela S, Väärt K, Niemelä M, Laakso A: Natural history of brain arteriovenous malformations: a long-term follow-up study of risk of hemorrhage in 238 patients. Neurosurgery 63:823-831, 2008

7. Heros RC, Korosue K, Diebold PM: Surgical excision of cerebral arteriovenous malformations: late results. Neurosurgery 26:570-578, 1990

8. Heros RC, Tu YK: Is surgical therapy needed for unruptured arteriovenous malformations? Neurology 37:279-286, 1987

9. Hoh BL, Kleinhenz DT, Chi YY, Mocco J, Barker FG II: Incidence of ventricular shunt placement for hydrocephalus with clipping versus coiling for ruptured and unruptured cerebral aneurysms in the Nationwide Inpatient Sample database: 2002 to 2007. World Neurosurg 76:548-554, 2011

10. Redekop G, TerBrugge K, Montanera W, Willinsky R: Arterial aneurysms associated with cerebral arteriovenous malformations: classification, incidence, and risk of hemorrhage. J Neurosurg 89:539-546, 1998

11. Spetzler RF, Martin NA: A proposed grading system for arteriovenous malformations. J Neurosurg 65:476-483, 1986

12. Stapf C, Mast H, Sciacca RR, Choi JH, Khaw AV, Connolly ES, et al: Predictors of hemorrhage in patients with untreated brain arteriovenous malformation. Neurology 66:1350-1355, 2006

Manuscript submitted October 31, 2012.

Accepted February 13, 2013.

Please include this information when citing this paper: DOI: 10.3171/2013.2.FOCUS12368.

Address correspondence to: Rose Du, M.D., Ph.D., Department of Neurological Surgery, Brigham and Women's Hospital and Harvard Medical School, 75 Francis Street, Boston, Massachusetts 02115. email: rdu@partners.org. 